\title{
Note on Coriolis-Stokes force and energy
}

\author{
Göran Broström • Kai H. Christensen • Magnus Drivdal • \\ Jan Erik H. Weber
}

Received: 15 October 2013 / Accepted: 17 April 2014 / Published online: 12 June 2014

(C) The Author(s) 2014. This article is published with open access at Springerlink.com

\begin{abstract}
In this study, we consider the origin of the CoriolisStokes (CS) force in the wave-averaged momentum and energy equations and make a short analysis of possible energy input to the ocean circulation (i.e., Eulerian mean velocity) from the CS force. Essentially, we find that the CS force appears naturally when considering vertically integrated quantities and that the CS force will not provide any energy input into the system for this case. However, by including the "Hasselmann force", we show some inconsistencies regarding the vertical structure of the CS force in the Eulerian framework and find that there is a distinct vertical structure of the energy input and that the net input strongly depends on whether the wave zone is included in the analysis or not. We therefore question the introduction of the "Hasselmann force" into the system of equations, as the CS force appears naturally in the vertically integrated equations or when Lagrangian vertical coordinates are used.
\end{abstract}

Keywords Energy $\cdot$ Coriolis-Stokes $\cdot$ Stokes drift $\cdot$ Eulerian · Lagrangian

Responsible Editor: Leo Oey

This article is part of the Topical Collection on the 5th International Workshop on Modelling the Ocean (IWMO) in Bergen, Norway 17-20 June 2013

G. Broström $(\bowtie)$

Department of Earth Sciences, University of Gothenburg,

Gothenburg, Sweden

e-mail: goran.brostrom@gu.se

K. H. Christensen • M. Drivdal

Norwegian Meteorological Institute, Oslo, Norway

J. E. H. Weber

Department of Geosciences, Oslo University, Oslo, Norway

\section{Introduction}

An object that moves in a rotating system will tend to veer relative the local frame due to inertia; in the rotating frame, the object appears to feel a force, and this is generally referred to as the Coriolis force. The traditional view is that the Coriolis force, which is always normal to the velocity, does not influence the energy budget as it is a consequence of inertia in a rotating system rather than being a true force. When waves are present in the rotating system, the analysis becomes less clear due to the uncertainty in how to treat the average of the fluctuating fields. A Lagrangian analysis shows that the Coriolis force will act on the mean drift of the waves (or the Stokes drift) and that the particles in a pure wave field will display horizontal orbits when averaged over the inertial period (Ursell 1950; Weber 1983). These studies show that the Stokes drift thus feels the Coriolis force in the same way as a traditional Eulerian current, and the force acting on the Stokes drift is now generally called the Coriolis-Stokes (CS) force. The influence of the CS force on the turbulent Ekman layer may be found in Polton et al. (2005). There have been a few recent studies where the energy input to the ocean circulation (i.e., Eulerian mean velocity) by the CS force have been addressed (Liu et al. 2007; Liu et al. 2009; Polton 2009; Wu and Liu 2008; Wu et al. 2008). In this study, we further investigate if the CS force can give rise to work done on the system. We argue here that earlier results indicating that the CS force influences the energy budget associated with the ocean circulation (i.e., the kinetic energy based on the Eulerian mean velocity) could possibly arise from a misrepresentation of the wave-drift and its forcing on the mean flow.

Any analysis of the wave-mean flow interaction has to deal with the fact that the sea surface, and fluid particles beneath the sea surface, oscillate. For instance, in a purely Eulerian view, the Stokes drift is zero in the main part of the fluid and appears in-between the wave trough and the wave crest. 
However, in a Lagrangian view, the Stokes drift in deep water decays continuously with depth as $\exp (2 k z)$, where $z$ is depth and $k$ is the wave number (Fig. 1). Thus, the two formulations give different results due to different treatment of the vertical level in the averaging process. It should be noted that averaging processes that are Eulerian-like but where the averaging process take Lagrangian motions into account give the Stokes drift with a vertical distribution equal to the purely Lagrangian analysis (Broström et al. 2008; Aiki and Greatbatch 2012; 2013). Another notion of interest here is that when considering the vertical integral of both the Eulerian and Lagrangian equations of motion, we regain consistency between the two formulations (Weber et al. 2006). The integral analysis of the wave influence on the mean flow carried out in Eulerian coordinates, as was developed by Longuet-Higgins and Stewart in a series of papers (Phillips 1977; Longuet-Higgins and Stewart 1960, 1961, 1962, 1964), provides a consistent description of the integrated properties of the wave-mean flow system. Furthermore, the integrated quantities represent one of the milestones regarding wave-mean flow interaction, and it is the framework on which most other theories are confirmed (Lane et al. 2007; Mellor 2013). However, it should be noted that this formulation does not provide any insight into the vertical structure of the wave-mean flow interactions. As a final notice, we would like to point out that the drift speed incorporating a Lagrangian Stokes drift is consistent with an observed trajectory movement (Röhrs et al. 2012).

\section{Basic theory}

We consider a simple case and thus neglect friction, assume an infinitely deep ocean, and consider an unforced system (i.e., the waves are steady in space and time). We also assume that mean currents are weak. Furthermore, we divide the total velocity, $\mathbf{u}=(u, v, w)$ which is in $\boldsymbol{x}=(x, y, z)$ direction and pressure, $p$, into Eulerian mean parts, $\mathbf{U}$ and $P$, and fluctuating parts, $\widetilde{\mathbf{u}}$ and $\widetilde{p}$ that will here represent wave fluctuations. Thus,

$\mathbf{u}=\mathbf{U}+\widetilde{\mathbf{u}}$

$p=P+\widetilde{p}$.

The analysis is based upon linearization of the governing equations, using the wave steepness, $\varepsilon_{k}=a k$, as a parameter of expansion ( $a$ is wave amplitude and $k$ is wave number). We will, in the following, assume that all wave quantities are $O\left(\varepsilon_{k}\right)$ while the mean flow is $O\left(\varepsilon_{k}^{2}\right)$. The equations to $O\left(\varepsilon_{k}\right)$ become

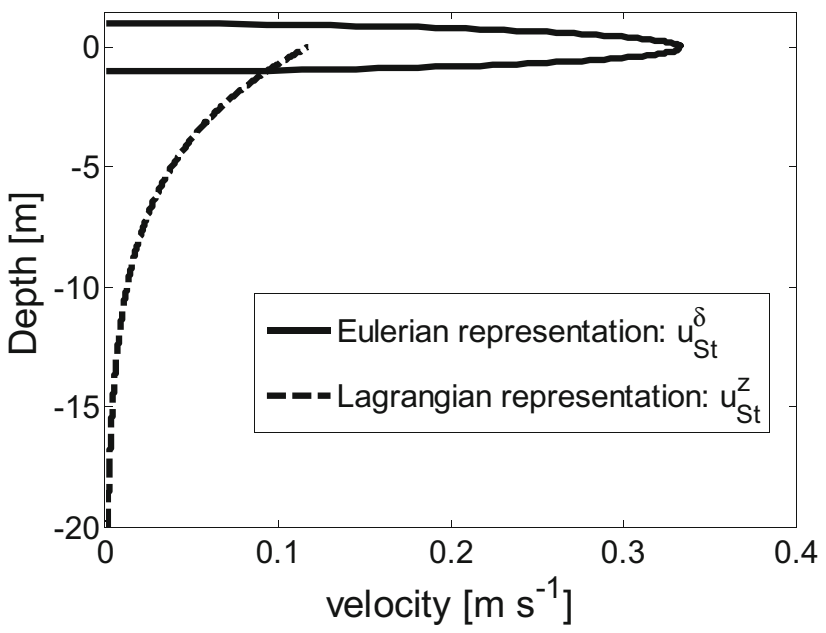

Fig. 1 Illustration of the Stokes drift in the Lagrangian and the Eulerian representations for a wave with amplitude $1 \mathrm{~m}$ and wave period $6 \mathrm{~s}$ in deep water. Here, we use $U_{S t}^{z}=a^{2} \omega k \exp (2 k z)$ and an approximate formula for the Eulerian flow, namely $U_{S t}^{\delta}=a \omega / \pi \sqrt{1-z^{2} / a}$. The total integral of the two representations are equal, but has different depth distributions

$\frac{\partial(U+\widetilde{u})}{\partial t}-f(V+\widetilde{v})+\frac{\partial \widetilde{u} \widetilde{u}}{\partial x}+\frac{\partial \widetilde{u} \widetilde{v}}{\partial y}+\frac{\partial \widetilde{u} \widetilde{w}}{\partial z}=-\frac{1}{\rho} \frac{\partial(P+\widetilde{p})}{\partial x}$,

$\frac{\partial(V+\widetilde{v})}{\partial t}+f(U+\widetilde{u})+\frac{\partial \widetilde{u v}}{\partial x}+\frac{\partial \widetilde{v v}}{\partial y}+\frac{\partial \widetilde{v} \widetilde{w}}{\partial z}=-\frac{1}{\rho} \frac{\partial(P+\widetilde{p})}{\partial y}$,

$\frac{\partial(W+\widetilde{w})}{\partial t}+\frac{\partial \widetilde{u} \widetilde{w}}{\partial x}+\frac{\partial \widetilde{w} \widetilde{w}}{\partial y}+\frac{\partial \widetilde{w} \widetilde{w}}{\partial z}=-\frac{1}{\rho} \frac{\partial(P+\widetilde{p})}{\partial z}-g$,

$\frac{\partial(U+\widetilde{u})}{\partial x}+\frac{\partial(V+\widetilde{v})}{\partial y}+\frac{\partial(W+\widetilde{w})}{\partial z}=0$,

where $f$ is the Coriolis parameter, $\rho$ is density, and $g$ the acceleration from gravity. Here, we include all wave-induced Reynolds stresses as many of them are important in wavemean flow interaction theories. As an example, $\frac{\widetilde{\partial u w}}{\partial z}$ is important when there are frictional boundary layers at the bottom or 
at the surface; in these cases, the term will represent the transfer of wave momentum to mean flow momentum in the Eulerian mean flow equation. The terms $\frac{\partial \widetilde{\partial u}}{\partial x}, \frac{1}{\rho} \frac{\partial p}{\partial x}$ will have non-zero values when integrated from the bottom to the undulating sea surface, and the combination of these (and the mean pressure from the undulating surface) is known as the wave radiation stress. Also, $\frac{\partial w w}{\partial z}$ represents a second-order pressure term, which appears in the expression for wave radiation stress. Finally, $\frac{\widehat{\partial v w}}{\partial z}$, which may (Hasselmann 1970), or may not, represent the CS force is the focus of this study; here, we refer to the force arising from this term as the "Hasselmann force".

We consider waves traveling in the $x$-direction, and, where we for the moment ignore the Coriolis force, find that the linear waves are described by

$$
\frac{\partial \widetilde{u}}{\partial t}=-\frac{1}{\rho} \frac{\partial \widetilde{p}}{\partial x}
$$

$\frac{\partial \widetilde{w}}{\partial t}=-\frac{1}{\rho} \frac{\partial \widetilde{p}}{\partial z}$,

$\frac{\partial \widetilde{u}}{\partial x}+\frac{\partial \widetilde{w}}{\partial x}=0$

For deep-water waves (extension to arbitrary depth is straightforward, but we consider deep-water waves for simplicity) with amplitude $a$, wave number $k$, and angular frequency $\omega$ (Phillips 1977),

$\widetilde{\zeta}=a \cos (k x-\omega t)$,

$\widetilde{u}=a \omega \exp (k z) \cos (k x-\omega t)$,

$\widetilde{w}=a \omega \exp (k z) \sin (k x-\omega t)$

$\widetilde{p}=\rho \frac{a \omega^{2}}{k} \exp (k z) \cos (k x-\omega t)$,

where $\widetilde{\zeta}$ is the fluctuating sea surface.

For waves on a rotating plane, it is sometimes considered that the resulting velocity in the $y$-direction, $\widetilde{v}$, may be described by (Xie et al. 2001; Hasselmann 1970)

$\frac{\partial \widetilde{v}}{\partial t}+f \widetilde{u}=0$

giving

$\widetilde{v}=a \omega \frac{f}{\omega} \exp (k z) \sin (k x-\omega t)$.
In practice, the inclusion of Eq. (5) in the analysis implies that we use a second small parameter of expansion, $\varepsilon_{f}=f / \omega$. Typically, $\varepsilon_{f}$ is very small, i.e., $\varepsilon_{f}<<\varepsilon_{k}$. From (6) and (2), we realize that the Coriolis force neglected in the $x$-component of (3) is $O\left(\varepsilon_{f}^{2}\right)$.

\subsection{Momentum equation}

To derive the wave forcing on the mean quantities, we need to insert the total velocity (and pressure) into the Navier-Stokes equations and take the mean over the wave fields to evaluate the forcing on the mean flow. However, this is not straightforward as the sea surface, as well as the particles in the fluid, oscillate vertically.

It is of some interest to see how the CS force appears in different settings. We ignore the second-order radiation stress and pressure terms as they have no significance in this analysis; however, we will keep the "Hasselmann force" $(\partial \widetilde{v} \widetilde{w} / \partial z)$. For an illustrative purpose, we keep all terms that are connected to $\widetilde{v}$. We arrive, after using Eqs. (3) and (5), at

$\frac{\partial U}{\partial t}-f(V+\widetilde{v})=0$,

$\frac{\partial V}{\partial t}+f(U+\widetilde{u})+\left(\frac{\partial \widetilde{v} \widetilde{w}}{\partial z}-f \widetilde{u}\right)=0$

Note that we connect $f \widetilde{u}$ from Eq. (6) with the "Hasselmann force", this is intentional as these two terms are both connected to each other by the inclusion of $\widetilde{v}$ in the analysis.

To find the wave forcing on the Eulerian mean flow, we need to take an average of the wave motions; in the following, we will consider two different averaging operators:

$\bar{a}=\frac{\omega}{2 \pi} \int_{0}^{2 \pi / \omega} a d t$,

$\langle a\rangle=\overline{\int_{-\infty}^{\zeta} a d z,}$

which represents a pure time mean (or Eulerian mean) and a time mean of the vertically integrated quantities. Here, it should be recalled that the Eulerian mean (8a) does not describe wave-mean flow interactions in a consistent way and does not provide a Stokes drift that is in agreement with, for example, a Lagrangian analysis. The integrated mean (8b) 
provides a well-established way to describe the entire forcing of the wave field on the mean flow, and it provides a correct description of the integrated Stokes drift. However, it does not provide any insight to the vertical distribution of the flow and the wave-mean flow interaction.

\subsubsection{Eulerian mean}

In the purely Eulerian mean (i.e., simple time average, Eq. 8a), we find for the different components in Eq. (7)

$\overline{f(V+\widetilde{v})}=f\left(V+V_{S t}^{\delta}\right)$

$\overline{f(U+\widetilde{u})}=f\left(U+U_{S t}^{\delta}\right)$

$\overline{\left(\frac{\partial \widetilde{v} \widetilde{w}}{\partial z}-f \widetilde{u}\right)}=f\left(U_{S t}^{z}-U_{S t}^{\delta}\right)$

where $U_{S t}^{\delta}$ represents the Stokes drift due to the Eulerian mean (at the surface or to be more precise over the area over which the surface fluctuates) and $U_{S t}^{z}$ the Lagrangian Stokes drift (distributed with depth). Their vertical distribution is displayed in Fig. 1. $V_{S t}^{\delta}$ is the Stokes drift in the $y$-direction (which, notably, is $\varepsilon_{f}$ smaller than the Stokes drift in the $x$ direction). The CS force according to the Hasselmann force expression is distributed as $\exp (2 k z)$, while the Coriolis force from the Stokes-drift term $(\widetilde{u})$ is exactly canceled out by $\overline{\partial \widetilde{v} / \partial t}$ However, it should be noted that the resulting CS force does not have the same depth distribution as the Stokes drift in the Eulerian frame which gives rise to an awkward inconsistency. If we, on the other hand, completely ignore the inclusion of $\widetilde{v}$, we find that the CS force has the same distribution as the Stokes mass flux, but that the vertical distribution is not consistent with the Lagrangian analysis; however, this is a well-known feature of the straightforward Eulerian mean.

\subsubsection{Integrated mean}

Validation of the integrated mean is a robust test of consistency since it includes the total momentum in the mean flow and the waves. Taking a vertical integral of the terms we investigate, followed by a time mean over the wave motions, give (note that $\widetilde{v}$ is out of phase with $\widetilde{\zeta}$, implying that the time mean of the product is zero)

$$
\overline{\int_{-\infty}^{\widetilde{\zeta}} f(V+\widetilde{v}) d z}=f\left(m_{y}\right)
$$

$$
\overline{\int_{-\infty}^{\widetilde{\zeta}} f(U+\widetilde{u}) d z}=f\left(m_{x}+m_{x}^{S t}\right)
$$

$$
\int_{-\infty}^{\widetilde{\zeta}}\left(\frac{\partial \widetilde{v} \widetilde{w}}{\partial z}-f \widetilde{u}\right) d z=f\left(m_{x}^{S t}-m_{x}^{S t}\right)=0
$$

where $m_{x}, m_{y}, m_{x}^{S t}$ are the total mean mass transport in $x-, y$ directions and the total Stokes drift (or Stokes flux) in $x$ direction, respectively. Now, we find that the CS force as arising from the Hasselmann force is canceled out exactly by $\langle\partial \widetilde{v} / \partial t\rangle$ and that the CS force that remains in the equations comes from the Coriolis force acting on the Stokes drift (i.e., it is derived from $\langle f \widetilde{u}\rangle$ ). Thus, for this case, the introduction of $\widetilde{v}$ in the analysis did not provide any new dynamics, implying that all relevant dynamics is present in the "standard" set $O\left(\varepsilon_{k}\right)$ wave equations in which Coriolis forces are ignored in the wave analysis.

\subsection{Energy equation}

To find the kinetic energy of the system, we multiply the $x$ momentum with $(U+\widetilde{u})$ and the $y$-momentum with $(V+\widetilde{v})$ and add the expressions. Continuing with our simple case and using Eq. (6), we find

$$
\begin{aligned}
(U+\widetilde{u}) \frac{\partial U}{\partial t} & -(U+\widetilde{u}) f(V+\widetilde{v})+(V+\widetilde{v}) \frac{\partial V}{\partial t}+(V+\widetilde{v}) f(U+\widetilde{u}) \\
& +(V+\widetilde{v})\left(\frac{\partial \widetilde{v}}{\partial t}+\frac{\partial \widetilde{v} \widetilde{v}}{\partial z}\right)=0
\end{aligned}
$$

The "standard" Coriolis terms cancel out exactly, the remaining terms are

$$
(U+\widetilde{u}) \frac{\partial U}{\partial t}+(V+\widetilde{v}) \frac{\partial V}{\partial t}+(V+\widetilde{v})\left(\frac{\partial \widetilde{v}}{\partial t}+\frac{\partial \widetilde{v} \widetilde{w}}{\partial z}\right)=0
$$

Thus, if we do not include $\widetilde{v}$ in the analysis (as is not necessary as shown earlier and will be further discussed in 
next section), the last term vanishes, and we find that the CS force will not influence the energy budget directly.

However, if we include $\widetilde{v}$ in the analysis, we have a contribution from the last term in Eq. (12). Since the Stokes drift is independent of time, we can write the time average of (12) as

$\frac{1}{2} \frac{\partial}{\partial t}\left[\left(U+U_{S t}^{\delta}\right)^{2}+\left(V+V_{S t}^{\delta}\right)^{2}\right]=-f V\left(u_{S t}^{Z}-u_{S t}^{\delta}\right)$.

The equivalent equation using vertical integration becomes (using 10 and 12)

$$
\int_{-D}^{\widetilde{\tau}} \frac{1}{2} \frac{\partial}{\partial t}\left[\left(U+U_{S t}^{\delta}\right)^{2}+\left(V+V_{S t}^{\delta}\right)^{2}\right] d z=-f V\left(m_{S t}^{x}-m_{S t}^{x}\right)=0
$$

Notably, by including $\widetilde{v}$ in the analysis, we find that the CS force will contribute to the energy budget, and we note that (1) it will have a distinct vertical dependence (2) the integrated mean of the contribution is quite small.

\section{Discussion and conclusion}

\subsection{Discussion on the CS force}

The starting point of this study was the question if the CS force could influence the energy budget of the mean flow. The answer to this question depends to some extent whether the mean kinetic energy is defined using the Eulerian mean velocity or the Lagrangian mean velocity. In many applications, the CS force has been derived using the Eulerian mean of the "Hasselmann" force $\partial \widetilde{v} \widetilde{w} / \partial z$. However, we again stress that an Eulerian mean of the Navier-Stokes equation has a rather complex physical interpretation and that a full account of the dynamics requires a separate treatment of the area between wave troughs and wave crests and the layer below the wave troughs (Newberger and Allen 2007a, b; Phillips 1977). Our analysis using an integrated framework, which describes the total momentum flux, shows that the CS force arises from the Stokes drift in the Coriolis term. Within this approach, the "Hasselmann" type of force is exactly zero as the time derivative of $\partial \widetilde{v} / \partial t$ exactly cancels out $\partial \widetilde{v} \widetilde{w} / \partial z$. Thus, introducing the small term $\widetilde{v}$ does not influence the dynamics, and we do not have to break the order of approximations (i.e., taking account of a term with magnitude $f / \omega$ while neglecting terms of order $\left.(a k)^{2} \gg f / \omega\right)$ that is the basis of wave and wave-mean flow interaction dynamics for deriving the CS force. The CS force has frequently been explained as a tilting of the planetary vorticity; however, this explanation is unnecessarily complicated. The CS force is simply the average Coriolis force that acts on a particle with a Lagrangian velocity as given by the mean currents and the waves. In the same way that the representation of the Stokes drift is problematic in an Eulerian reference frame, there is a problem of representing the CS force.

\subsection{Vertical structure of the CS force}

So far, we have only considered the Eulerian mean and the integrated mean. However, the integrated framework does not provide insight into the vertical structure, and the Eulerian analysis raises the question if there is vertical dependence not seen in the integrated analysis. One way to analyze the vertical structure is to use a quasi-Lagrangian, quasi-Eulerian representation (Broström et al 2008). Hence, we use an averaging operator defined as

$\{a\}=\frac{\partial}{\partial Z} \int_{-\infty}^{Z+\tilde{\zeta}_{Z}} a d z$

which represents the time mean of an infinitesimal thin oscillating layer located at a mean level $Z$ and where the fluctuation is given by

$\widetilde{\zeta}_{z}=\int \widetilde{w} d t$

Using this formalism, we find

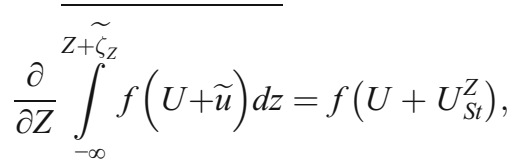

$$
\frac{\partial}{\partial Z} \int_{-\infty}^{\overline{Z+\tilde{\zeta}_{Z}}\left(\frac{\partial \widetilde{v}}{\partial z}-f \widetilde{u}\right) d z}=f\left(U_{S t}^{Z}-U_{S t}^{Z}\right)=0
$$

Thus, this result is consistent with the result from the integrated mean. The CS force comes from the Coriolis force acting on the Stokes drift, and the contribution from including $\widetilde{v}$ into the system is exactly canceled out. This statement is approximately valid for the energy analysis as well (the case 
with strongly sheared flow may, however, give non-negligible contributions from the "Hasselmann" type of force). It should be noted that this formalism provides, by definition, exactly the same result as the integrated framework in case we integrate over the entire fluid.

\subsection{General discussion on CS force and energy}

There have been a few recent studies suggesting that the CS force may contribute to the budget of the kinetic energy which is defined using the Eulerian mean velocity (Liu et al. 2007; Liu et al. 2009; Polton 2009; Wu and Liu 2008; Wu et al. 2008). This would be a surprising result as the traditional Coriolis force does not influence the budget of the kinetic energy which is defined using raw velocity as it is a "virtual" force rather than a true force. The basis of earlier studies is that the "Hasselmann" force actually does influence the mean energy equation in an Eulerian framework; however, it is well-known that the truly Eulerian framework cannot describe the wave-mean flow interaction in a consistent way. An alternative, more correct, description is to integrate all equations from the bottom to the sea surface. This formulation does not provide any information on the vertical distribution of the induced mass transports or the wave-mean flow interaction, but it is generally considered to be a solid ground truth for wave-mean flow interaction. Providing consistency with the integrated quantities is often considered an essential test case for new developments in wave-mean flow interaction theories (Lane et al. 2007; Mellor 2013).

In this study, we show that the CS force does not influence the mean-flow energy budget when a proper treatment of the entire wave field is considered. Furthermore, using integration to an imaginary marked layer, we find that the CS force does not influence the mean energy at any depth (within the order of approximations); notably, this formulation provides a realistic depth distribution of the Stokes drift (Broström et al 2008). This result is consistent with our basic understanding that the Coriolis force, and its wave counterpart the CS force, is a virtual force arising from inertia in a rotating system.

\subsection{Indirect influence of CS on energy}

We conclude that the CS force does not have a direct impact on the budget of the kinetic energy which is defined using the Lagrangian mean velocity. However, as it may influence the direction of the surface current, it can have an indirect impact on the total kinetic input to the system. Following Philips (1977), the energy input, say $W$, into the system is

$W=\mathbf{U} \cdot \boldsymbol{\tau}$,

where $\boldsymbol{U}$ is the mean Eulerian velocity at the surface, and $\tau$ is the momentum flux from the atmosphere into the ocean.

To get an idea how this term changes when the CS force is included, we consider a one-dimensional mixing model for Ocean Weather Station M (located at 66 N, 2 E) for the year 1987. We use the General Ocean Turbulence Model (GOTM, Umlauf and Burchard 2005, 2003) and run the model with (1) standard configuration and (2) for a case where CS force is included. Wave data are obtained from the ERA-Interim reanalysis (Dee et al. 2011). The results are shown in Fig. 2. We see that the sea surface temperature (SST) becomes slightly lower when including the CS force, which indicates that the CS force does alter the energy available for mixing. The CS force veers the wind-driven current, thus the energy input (18) becomes different. The small impact on SST does, however, indicate that the effect is not large.
Fig. 2 The sea surface temperature at Ocean Weather Station M for 1987. The figure shows two different runs using the GOTM turbulence model: a reference run (blue), and a run that includes the CS force (green). The difference in SST between the two runs is about $0.1^{\circ} \mathrm{C}$ during summer

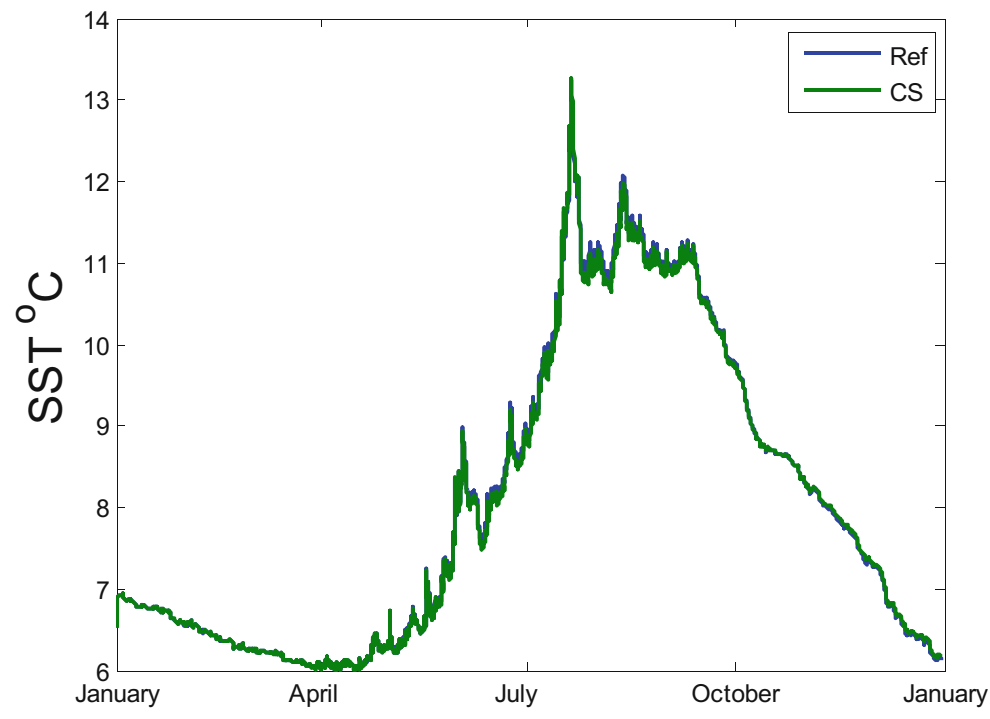




\subsection{Conclusion}

We have shown that the CS force can be derived in two ways, one direct way where the wave perturbations are evaluated using an integrated framework and by including the wave motion that result from the Coriolis force. We consider the first method to be more consistent with results from Lagrangian analysis and we suggest that Lagrangian-like averages such as Genreralized Lagrangian Mean (GLM) (Ardhuin et al. 2008; Bennis and Ardhuin 2012; Bennis et al. 2011) or frameworks based on integrated means (Aiki and Greatbatch 2012, 2013; Broström et al. 2008) are considered when deriving equations describing the wave-mean flow interactions. We note that the exact way of deriving the CS force may have an influence of the estimate of the energy budget of the upper ocean. By including the "Hasselmann" type of force, we find that the CS force, in an Eulerian setting, will contribute directly to the energy budget of the upper ocean. This shows an inconsistency toward the integrated analysis, and this route to deriving the CS force should therefore be avoided.

Acknowledgments We gratefully acknowledge financial support from the Research Council of Norway through the grants 196438 (BioWave) and 207541 (OilWave). Three anonymous reviewers also contributed to the final version of the manuscript.

Open Access This article is distributed under the terms of the Creative Commons Attribution License which permits any use, distribution, and reproduction in any medium, provided the original author(s) and the source are credited.

\section{References}

Aiki H, Greatbatch RJ (2012) Thickness-weighted mean theory for the effect of surface gravity waves on mean flows in the upper ocean. J Phys Oceanogr 42(5):725-747. doi:10.1175/Jpo-D-11-095.1

Aiki H, Greatbatch RJ (2013) The vertical structure of the surface wave radiation stress for circulation over a sloping bottom as given by thickness-weighted-mean theory. J Phys Oceanogr 43(1):149-164. doi:10.1175/Jpo-D-12-059.1

Ardhuin F, Rascale N, Belibassakis KA (2008) Explicit wave-averaged primitive equations using a Generalized Lagrangian Mean. Ocean Model 20:235-264

Bennis A-C, Ardhuin F (2012) Comments on "The depth-dependent current and wave interaction equations: a revision". J Phys Oceanogr 41:2008-2012

Bennis A-C, Ardhuin F, Dumas F (2011) On the coupling of wave and threedimensional circulation models: choice of theoretical framework, practical implementation and adiabatic tests. Ocean Model 40:260-272

Broström G, Christensen KH, Weber JE (2008) A quasi-Eulerian quasiLagrangian view of surface wave induced flow in the ocean. J Phys Oceanogr 38(5):112-1130

Dee DP, Uppala SM, Simmons AJ, Berrisford P, Poli P, Kobayashi S, Andrae U, Balmaseda MA, Balsamo G, Bauer P, Bechtold P, Beljaars ACM, van de Berg L, Bidlot J, Bormann N, Delsol C, Dragani R, Fuentes M, Geer AJ, Haimberger L, Healy SB, Hersbach H, Holm EV, Isaksen L, Kallberg P, Kohler M, Matricardi M, McNally AP, Monge-Sanz BM, Morcrette JJ, Park BK, Peubey C, de Rosnay P,
Tavolato C, Thepaut JN, Vitart F (2011) The ERA-Interim reanalysis: configuration and performance of the data assimilation system. Q J Roy Meteor Soc 137(656):553-597. doi:10.1002/Qj.828

Hasselmann K (1970) Wave-driven inertial oscillations. Geophys Fluid Dyn 1:463-502

Lane E, Restrepo JM, McWilliams JC (2007) Wave-current interaction: a comparison of radiation-stress and vortex-force representations. J Phys Oceanogr 37:1133-1141

Liu B, Wu K, Guan C (2007) Global estimates of wind energy input to subinertial motions in the Ekman-Stokes Layer. J Oceanogr 63:457-466

Liu B, Wu K, Guan C (2009) Wind energy input to the Ekman-Stokes layer: reply to comment by Jeff A. Polton. J Oceanogr 65:669-673

Longuet-Higgins MS, Stewart RW (1960) Changes in the form of short gravity waves on long waves and tidal currents. J Fluid Mech 8:565-583

Longuet-Higgins MS, Stewart RW (1961) The changes in amplitude of short gravity waves on steady non-uniform currents. J Fluid Mech 10:529-549

Longuet-Higgins MS, Stewart RW (1962) Radiation stress and mass transport in gravity waves, with application to 'surf beats'. J Fluid Mech 13:481-504

Longuet-Higgins MS, Stewart RW (1964) Radiation stress in water waves; a physical discussion with applications. Deep-Sea Res 11:529-562

Mellor G (2013) Waves, circulation and vertical dependence. Ocean Dyn 63(4):447-457. doi:10.1007/s10236-013-0601-9

Newberger PA, Allen JS (2007a) Forcing a three-dimensional, hydrostatic, primitive-equation model for application in the surf zone: 1 . Formulation. J Geophys Res Oceans 112, C8. doi:10.1029/ 2006jc003472

Newberger PA, Allen JS (2007b) Forcing a three-dimensional, hydrostatic, primitive-equation model for application in the surf zone: 2 . Application to DUCK94. J Geophys Res Oceans 112, C8. doi:10. 1029/2006jc003474

Phillips OM (1977) The dynamics of the upper ocean. Cambridge University Press, Cambridge

Polton JF (2009) A wave averaged energy equation: comment on "Global estimates of wind energy input to subinertial motions in the EkmanStokes Layer" by Bin Liu, Keijian Wu and Changlong Guan. J Oceanogr 65:665-668

Polton JA, Lewis DM, Belcher SE (2005) The role of wave-induced Coriolis-Stokes forcing on the wind-driven mixed layer. J Phys Oceanogr 34(4):444-457

Röhrs J, Christensen K, Hole LR, Broström G, Drivdal M, Sundby S (2012) Observation based evaluation of surface wave effects on trajectory forecasts. Ocean Dyn 62(10-12):1519-1533

Umlauf L, Burchard H (2003) A generic length-scale equation for geophysical turbulence models. J Mar Res 61(2):235-265. doi:10.1357/ 002224003322005087

Umlauf L, Burchard H (2005) Second-order turbulence closure models for geophysical boundary layers. A review of recent work. Cont Shelf Res 25(7-8):795-827. doi:10.1016/j.csr.2004.08.004

Ursell F (1950) On the theoretical form of ocean swell on a rotating earth. Mon Not R Astron Soc, Geophys Suppl 6:1-8

Weber JE (1983) Steady wind- and wave-induced currents in the open ocean. J Phys Oceanogr 13(3):524-530

Weber JE, Broström G, Sætra Ø (2006) Eulerian vs Lagrangian approaches to the wave-induced transport in the upper ocean. J Phys Oceanogr 36(11):2106-2118

Wu K, Liu B (2008) Stokes drift-induced and direct wind energy inputs into the Ekman layer within the Antarctic circumpolar current. J Geophys Res 113, C10002. doi:10.1029/2007JC004579

Wu K, Yang Z, Liu B, Guan C (2008) Wave energy input into the Ekman layer. Sci China Ser D Earth Sci 51(1):134-141

Xie L, Wu K, Pietrafesa L, Zhang C (2001) A numerical study of wavecurrent interaction through surface and bottom stresses: wind-driven circulation in the South Atlantic Bight under uniform winds. J Geophys Res C Oceans 106(C8):16,841-16,855 